\title{
35
}

\section{Hypermedia for open and flexible learning}

\author{
K. Hendrikx \\ E. Duval \\ H. Olivié
}

Katholieke Universiteit Leuven

Belgium

\begin{abstract}
Although there are many systems available for the creation and maintenance of course material for open and flexible learning, this task remains tedious and inefficient. In this paper we present a data model and a design paradigm for hypermedia courseware. We demonstrate an implementation of the model which makes use of the World Wide Web, and will be used to provide selfstudy courses to students of the university of Leuven.
\end{abstract}

Main conference themes: flexible learning, distance learning

Educational areas: higher education

Study topics:

Secondary keywords: courseware, hypermedia, networks 


\section{BACKGROUND}

\section{A modular self study course}

At the Katholieke Universiteit Leuven a course introducing basic concepts of computer science is included in the curriculum of students in law, medicine, economics, arts and social sciences. We are transforming this course into a modular self study course for of two reasons :

- The individual students have diverse backgrounds: some have extensive computer experience, while others have no such experience at all. As pretests can determine whether or not a module is necessary for a particular student, the course can be individualized.

- Although there is a significant overlap between the basic computer science courses in the different faculties, the courses are not identical. The modular structure of the self study course will enable each faculty to organize the course according to its needs:

- relevant modules can be selected from a common pool of modules;

- specific topics, of interest to a specific faculty, can be elaborated in specific modules.

\section{THE USE OF HYPERMEDIA IN EDUCATION}

In order to handle hypermedia resources for education (text, images, animations, interactive tests) we rely on a hypermedia data model. The choice of a good data model is essential in the context of a large scale hypermedia system because one can get lost in hyperspace and because of fragmentation problems [1].

Traditional data models (hierarchical, network or relational) from the field of databases are quite effective to model structured data [2]. These are however not very well suited for modelling the unstructured and complex data which characterize multimedia and hypermedia environments. Special purpose data models have therefore been developed for this application area.

A hypermedia data model is a more or less formal approach to describe properties of hypermedia environments. The simplest hypertext model is based on a directed graph of information chunks called nodes, where links represent the simple relationships between the nodes $[1,3,4]$. This model is called the node-link paradigm or the basic hypermedia paradigm [5]. Associated with this model is a default browsing semantics: any link emanating from a node can be selected in order to navigate to its destination. 
Without additional structuring mechanisms the flatness of the basic hypermedia paradigm results in a number of problems with large scale hypermedia networks which can be summed up as follows:

- hard to manage for designers, because insertion of new nodes requires updating of links;

- difficult to understand for readers.

Also modularization of design and reuse of resources is difficult to achieve in the basic hypertext model.

More recent models have added a number of constructs to the basic paradigm in order to make it more flexible, to increase its modelling expressiveness and to overcome what has been called 'the tyranny of the link':

- Composite nodes themselves are graphs of nodes and links, and represent hierarchical organization of information. A collection of nodes is thus abstracted into one higher order object [3]. In [6] it was proposed that composites should be elevated to peer status with atomic nodes and links. A similar appeal was made in [7] on the basis of comparison of links to go-to statements in programming languages.

- Other models are based on sets as a hypermedia modelling construct, e.g. the HM Data Model [2].

- In [8] Petri-nets are proposed as a basis for hypermedia modelling.

- Virtual structures may be defined by computational procedure rather than by explicit specification of their components [3]; examples include navigation by query [9], implicit links from a word to its entry in a dictionary [10], etc.

\section{A HYPERMEDIA DATA MODEL}

\section{A model for learning material}

Our data model is a variation of the $\mathrm{HM}$ data model [2]. This model adheres to an object oriented approach for modelling hypermedia data. All data are encapsulated in so-called S-collections (structured collections). S-collections may contain a number of other S-collections, called members. This hierarchical structure adds an extra dimension to hypermedia, namely that of scale. Besides following links between S-collections users can zoom in and out of Scollections.

Five subclasses are derived from the class S-collection. The subclasses correspond to different link topologies:

- An S-collection of type VOID has no further internal structure; it simply acts as a container for multimedia information. 
- In a MENU one member is the head. All other members are linked to the head, and vice versa.

- In an ENVELOPE each member is linked to every other member.

- In a FOLDER the members are ordered and each member is linked to a previous and next member.

- In a FREELINKS collection arbitrary links can be defined between members.

Each class of S-collection has its own ACCESS method. When an S-collection of type VOID is accessed, its contents are displayed. When any other Scollection is accessed, its head member is accessed (and so on until a VOID receives the access message and displays its contents) and a number of links becomes available. Activation of a link results in access to the S-collection linked to.

Links are stored separately from the content hypermedia documents. These are always local to an S-collection. This makes it possible to reuse Scollections in other contexts without modification. Each S-collection can be a member of more than one other S-collection.

S-collections are not necessarily static. These may be generated by querying the database of S-collections or by a software process invoked at the time of access. This for instance makes it possible to define an S-collection which contains 'the most recent satellite picture of this area' or 'ten random true-false questions on the history of computing'.

\section{AN EXTENSION FOR EDUCATION}

\section{Modelling the learner}

We have added two functions to the HM data model in order to make it more suitable for application in an educational setting.

Accessed(c:S-collection, u:user):Boolean is true when user $\mathbf{u}$ has accessed the S-collection c, and false otherwise.

Completed(c:S-collection, u:user):Boolean is true when user $\mathbf{u}$ has completed S-collection c.

For an S-collection of type VOID the function Completed equals the function Accessed. For other S-collections its value depends on extra type attributes. These type attributes may be one or more of the following:

- An S-collection of type TEST will return true for the function Completed only if the user has passed the test. The software implementing the test must also ensure that a score database is updated when the user has completed the test. 
- If an S-collection is marked MULTIPLEXED, the function Completed will return true if at least one of its members is completed. Any other Scollection is completed when all its members are completed.

- An S-collection marked OPTIONAL will always return true for the function Completed.

These types define the function Completed. Two other types are used to limit a user's freedom in accessing S-collections:

- An S-collection marked VIRGIN may only be accessed once by a certain user. This is useful for tests which the user may only try once.

- In an S-collection marked ORDERED a user may only access a certain member of that S-collection if Completed is true for all previous members.

In this context the course we are developing would be defined as an ENVELOPE or a FOLDER containing two members. The first (head) is the introduction and the second the content. Our particular course is called bmi (Fig. 1).

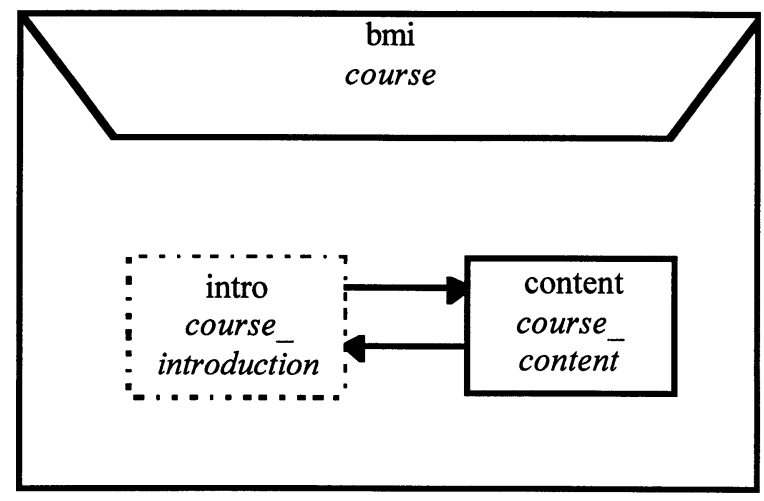

Fig. 1 The course is an ENVELOPE

The course introduction is a MENU of which the head is a VOID S-collection containing a short introduction to the course. Its members are OPTIONAL VOID S-collections which appear again inside the course content. This gives the student the option of browsing through each module's introduction before actually starting the course (Fig. 2).

The course content is defined as an ORDERED FOLDER of $\mathrm{S}$ collections (Fig. 3). The fact that it is ORDERED implies that the students must complete the modules in the given order. 
Each module in turn is a MULTIPLEXED MENU with three members. The head which is the module's introduction, the pretest and the content (Fig. 4). The pretest is VIRGIN TEST so that the user may only try it once.

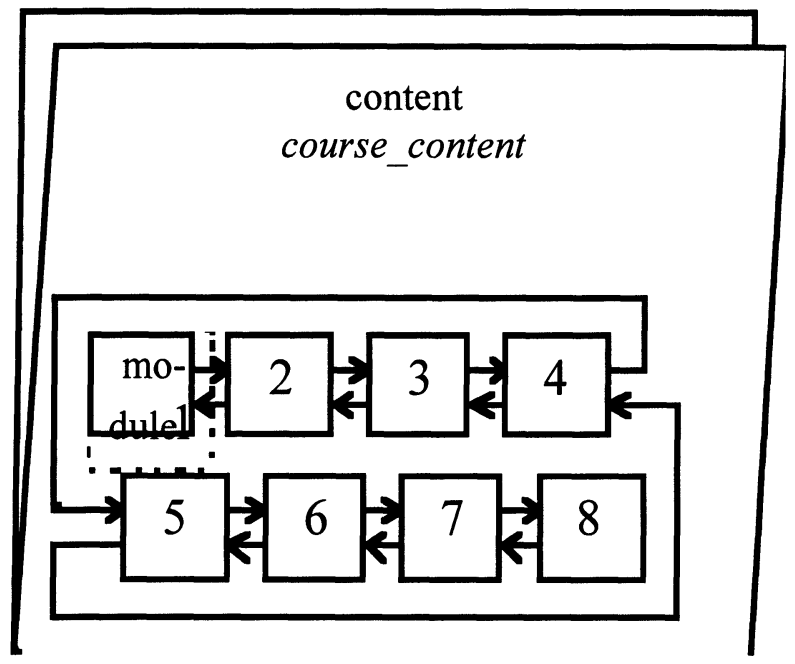

Fig. 2 The course content is a FOLDER

Thus, when a student accesses a module, its introduction is displayed (some text describing the module). Two options are available to the student: either to do the pretest or to go to the module's content. If the pretest is successfully completed, access is allowed to the next module of the course, since the module is now completed. Otherwise the module's content will have to be completed again maybe consisting of a number of S-collections possibly with intermediate tests, assignments and containing a post-test.

\section{QUERYING MECHANISMS AND STRUCTURAL CONSTRAINTS}

\section{Instruments for teachers and learners}

Additional properties can be assigned to S-collections such as a category name and structural constraints. A constraint could for instance be the following: a certain category of S-collections may only contain S-collections out of some other specific category. We are developing a framework which will allow queries to use this structural information. For example, we may define the category course as a FOLDER containing two members. The first is an Scollection of category course_introduction and the second is of category 
course_content (Fig. 1). The course_content is defined as an ORDERED FOLDER of S-collections of category module (Fig. 3). Similarly a module can be constrained to be a MULTIPLEXED MENU with three members: the first of category module_introduction, the second of category pretest and the third of category module_content (Fig. 4).

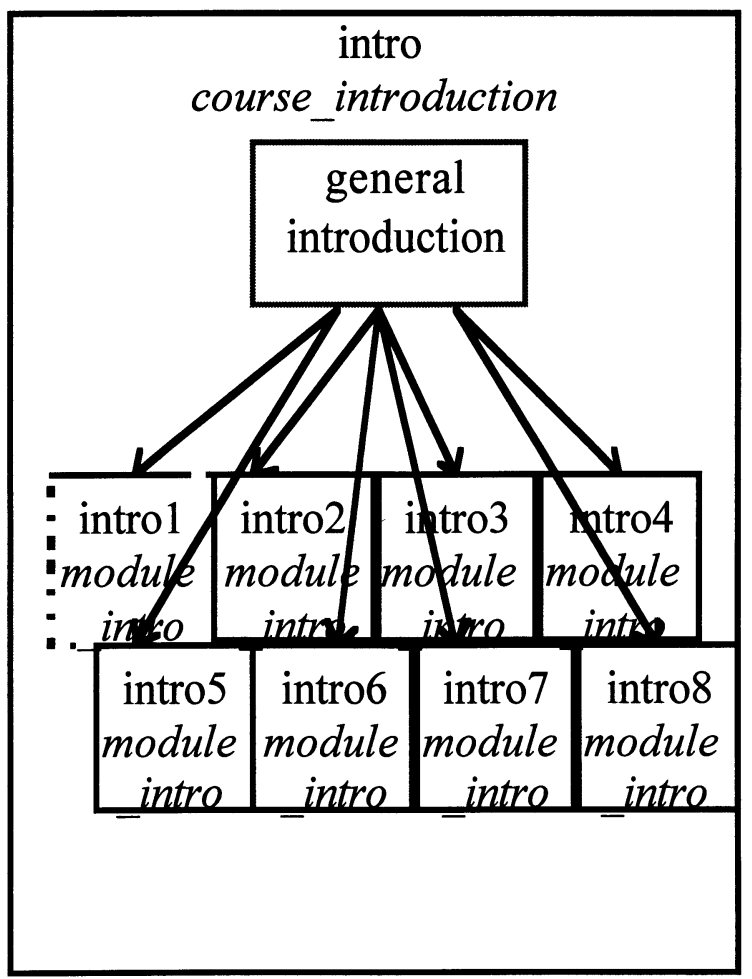

Fig. 3 The introduction

A query could be used to create dynamic S-collections. Suppose we want to create an automatic overview of all existing modules. For this a new Scollection could be created, e.g. a MENU, containing all S-collections which belong to the category module_introduction. 


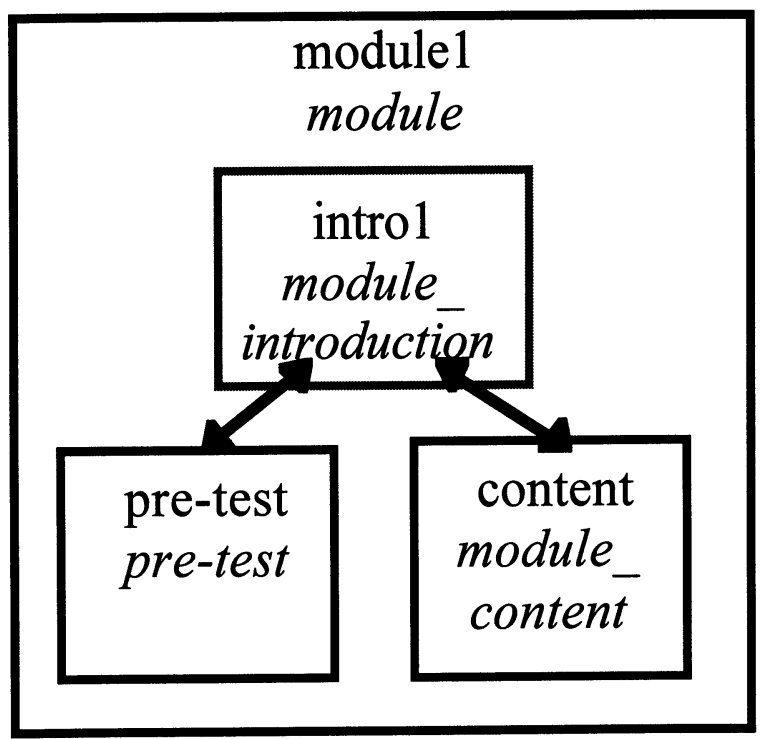

Fig. 4 Each module is a MULTIPLEXED MENU

\section{AUTHORING AND MANAGEMENT OF LEARNING RESOURCES}

\section{What this model can do for you}

The above data model is a sound basis for hypermedia courseware and offers a number of important advantages for authoring and managing hypermedia learning material, especially when large amounts of resources are concerned.

\section{Structured (top-down) design of hypermedia courses}

The hierarchical nature of S-collections allows the author to design a course in a top-down fashion, much as in the example given above. Alternatively, a bottom-up approach can be used: starting from the multimedia resources (texts, images, tests, etc.) small S-collections can be created. These can later be grouped into larger S-collections.

S-collections thus add an extra level of abstraction to the design of hypermedia courseware. Problems with manual link editing which arise when new documents are created and old ones are deleted (dangling references), are eliminated. 


\section{Reuse of learning resources}

Each S-collection in itself is a selfcontained unit. The knowledge or skills which the student will have acquired when it is completed, can be defined explicitly. This notion of selfcontainment is essential in order to support large scale reuse of resources.

The Completed function effectively models the student's knowledge since it describes which S-collections he or she has successfully completed.

\section{Separation of structure and content}

Because the hypermedia data itself and the structure imposed on it by the Scollections are stored separately, the structure of a course and its actual content are effectively separated. This makes it possible to first design the structure and add the content later which is a form of top-down design.

This approach also makes it possible to impose different structures on a particular content (e.g. a version of a course without the tests in which students can browse through the course, or a version accompanying a book with demonstrations and tests only) or use different contents in a particular structure (e.g. versions in different languages). It is also possible to store the content on read-only storage (CD-ROM) and still be able to edit the structure.

\section{Dynamic S-collections can provide individual guidance to learners}

Attributes can qualify the instructional content or pedagogical function of Scollections. Remediation can then take the form of a query. Once a test has established which skills or knowledge a student has acquired and which not, a query can be formulated which will generate an S-collection constituting a personalized learning scenario.

\section{AN IMPLEMENTATION USING THE WORLD WIDE WEB}

\section{How we did it}

We are developing an introductory self study course on the subject of computer science using the methods described above. As a development platform we used the Word Wide Web (WWW). The WWW is a client-server based hypermedia system. The client displays hypermedia documents and acts as a user interface. The server stores the documents and delivers them to the client. Client and server may run on different computers in a networked environment. Because the WWW is such an open and flexible system, it has allowed us to implement our own system.

A database with information about students, descriptions of S-collections and the hypermedia resources is managed by the server. When a link is 
activated at a URL (Uniform Resource Locator), it is sent back by the client program and is mapped onto an S-collection. Accessing the S-collection generates a page of HTML (HyperText Mark-up Language, the format used by the WWW) which is sent back to the client to be displayed.

In addition we have developed an Application Programmer Interface (API) to allow existing or new courseware to be used as hypermedia learning resources in our environment. For example, an interactive test or demonstration can be programmed in any authoring language such as IconAuthor or Authorware. The API can be used to communicate the score to the server.

The system is based on a layered design. Figure 5 illustrates the layers and the flow of data.

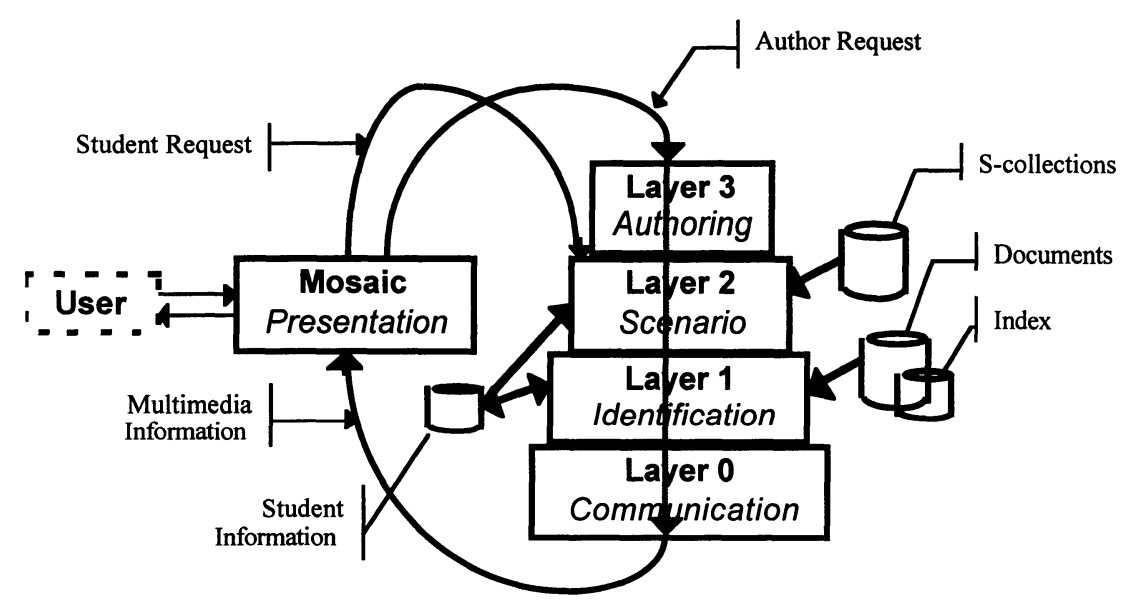

Fig. 5 A schematic view of the layers and the data flow.

\section{Level 0 : Communication and Presentation}

Layer 0 is the WWW software which takes care of the communication and presentation aspects. For the presentation the well-known client Mosaic is used.

A practical problem with this layer is that documents and hypertext links correspond to physical files. This has the following disadvantages:

- The granularity of text has to be defined at the lowest level and later modification is very difficult. Also, maintaining a large number of small files is impractical. 
- Using file names in hypertext links results in a rather low level implementation of courseware which makes maintenance very difficult. If a file is moved or renamed, all references to that file would have to be changed.

For these reasons the Layer 1 was introduced.

\section{Layer 1 : Identification}

This layer separates the identification of hypermedia objects from the underlying file structure. It keeps an index of hierarchical resource names in relation to a file or part of a file. For instance, the name bmi identifies everything related to the basic computer science modules. The name

\section{bmi.module1.virtual_machine.definition}

identifies a small paragraph of text which defines the concept of a virtual machine. These hierarchical resource names may identify resources other than text. For instance, the name

\section{bmi.module1.virtual_machine.example.demo}

is an interactive demonstration of a virtual machine written in the authoring language IconAuthor.

Other tasks of the identification layer are:

- indexing: the user can browse through the hierarchical index or search for keywords. The index also contains a short description of the content for each entry.

- managing annotations: the server can show personal annotations for each resource. Annotations are stored as a difference between the original text and the annotated text so that a user can actually edit a text without changing the original. All students can add own notes to the text or mark words. Annotations can be made visible to a group of users.

\section{Layer 2 : The scenario layer}

The scenario layer implements the S-collection class and its derived classes as described earlier.

\section{Layer 3 : The authoring layer}

This layer is only accessible to more privileged users. It enables teachers to create new S-collections and to edit existing ones.

It also produces statistics on student performance (their scores on different tests, the information they accessed, etc.). With this information the teaching staff can evaluate the course and improve those parts which have proved problematic at tests. 
This layer also gives access to a database of questions and tests which offers support for automatically generating a test about a certain subject.

\section{CONCLUSION}

In this paper we have described a design and implementation paradigm for hypermedia courseware. This paradigm is both simple and powerful, and it has numerous advantages especially with respect to maintainability.

It can also be extended to allow dynamic structures, querying and the use of structural constraints. However, more research is required here.

\section{REFERENCES}

1. Conklin, J. (1987) Hypertext: An Introduction and Survey. IEEE Computer, 20, pp. 17-41.

2. Maurer, H., Scherbakov, N. and Srinivasan, P. (1993 A new Hypermedia Data Model, in Proceedings of DEXA'93, 4th International Conference on Database and Expert Systems Applications, Prague, Czech Republic, Lecture Notes in Computer Science 720, Springer, pp. 685-696.

3. De Bra, P. Houben, G.-J. and Kornatzky, Y. (1992) An Extensible Data Model for Hyperdocuments, in ECHT'92, pp. 222-231.

4. Nielsen, J. (1990) Hypertext and Hypermedia. Academic Press.

5. Maurer, H., Andrews, K. and Scherbakov, N. (1994) Semantic Modelling of Object-Oriented Hypermedia Databases. Accepted for DEXA'94.

6. Halasz, F. (1988) Reflections on NoteCards: Seven issues for the next generation of hypermedia systems. CACM, 31 (7) pp. 836-852.

7. van Dam, A. Hypertext'87: Keynote Address. CACM, 31 (7) pp. 887-895.

8. Stotts, P. D., Furuta, R. (1989) Petri-net-based hypertext: document structure with browsing semantics. ACM Transactions on Information Systems, 7 (1) pp. 3-29. 
9. Tanaka, K., Nishikawa, N., Hirayama, S. and K. Namba, K. (1991) Query pairs as hypertext links, in Proceedings of the IEEE Data Engineering Conference, pp. 456-463.

10. Yankelovitch, N., Haan, B., Meyrowitz, N. and Drucker, S. (1988) Intermedia: the concept and the construction of a seamless information environment. IEEE Computer, 21 (1) pp. 81-96. 